\title{
Collagen Structure: Evidence for a Helical Organization of the Collagen Fibril
}

\author{
John H. Lillie, ${ }^{1}$ Donald K. MacCallum, ${ }^{1}$ Lawrence J. Scaletta, ${ }^{2}$ \\ AND Joseph C. OCCHINO ${ }^{2}$ \\ 'Dental Research Institute and Department of Anatomy, Medical School, University of Michigan, Ann \\ Arbor, Michigan 48109; and ${ }^{2}$ Department of Oral Biology, School of Dentistry, Case Western Reserve \\ University, Cleveland, Ohio 44106
}

Received May 7, 1976, and in revised form, August 17, 1976

\begin{abstract}
The collagen fibrils of human or guinea pig dermis when exposed to the denaturing agents, urea or guanidine- $\mathrm{HCl}$, dissociated into smaller, disparate subunits, probably aggregates of microfibrils. The process of dissociation demonstrates that the fibrils are assembled helically. Initially, diagonal clefts appear on the surface of the fibril. These clefts are surface manifestations of a spirally oriented, internal space. Continued exposure to these denaturants resulted in progressive dissociation of the fibril into helically oriented subunits. It is suggested that water-miscible compounds such as glycols or hydroxypropyl methacrylate, in addition to the urea-guanidinium class of denaturants used in this study, affect the observed fbrillar changes through the disruption of hydrogen bonds between the microfibrils making up the fibril. Such a mode of action may explain why freeze-fractured or "inert embedded" collagen demonstrates helical organization while other, more conventional methods of tissue processing do not. Further support for the proposed mode of action of these dissociative agents was provided by the observation that mature collagen, in which extensive intra- and intermolecular covalent crosslinks are present, is more resistant to dissociation than newly formed collagen.
\end{abstract}

The now classical ultrastructural description of collagen as a fibril demonstrating $64 \mathrm{~nm}$, periodic cross banding due to the precise ordering of the substituent molecules, stands as one of the few examples in biology where the appearance of a fixed, sectioned and stained specimen can be reasonably well explained on the basis of its molecular structure. However, the intermediate (supramolecular) levels of fibril organization, those between the collagen molecule and the fibril proper, have only recently begun to be explored. It is currently believed that collagen molecules are assembled into microfibrils, which in cross section are made up of between 4 and 8 collagen molecules $(7,11,22)$, and that these microfibrils are in turn assembled into fibrils. Another intermediate unit of organization, the subfibril, is present in reconstituted collagen fibrils; however, there is no current evidence for its existence in native collagen (7).

A recent review has emphasized the bio- chemical and biophysical evidence for coiling in three (polypeptide chain, collagen molecule, microfibril) of the four levels of fibril organization, the last level of organization being the fibril proper (18). Morphological studies of collagen prepared by freeze-fracture procedures have described diagonal clefts in fibrils which suggest that the fibril itself is helically organized $(4,19,23)$. These recent freeze-fracture studies have rekindled interest in earlier observations made on negatively stained (or shadow casted) intact fibrils or embedded and sectioned fibrils which suggested that the fibril was assembled helically ( 3 , $6,18,25)$. In this report, we describe a helical type of organization present in dermal collagen fibrils subsequent to exposure of the tissues to dissociative solvents. We further propose a mechanism which may explain why certain preparative procedures demonstrate the helical organization of fibrils while other, more conventional methods do not. 


\section{MATERIALS AND METHODS}

Specimen preparation. Samples of human and guinea pig dermis were used for these studies. Human samples were obtained from the abdominal walls of nine patients at autopsy. The individuals studied ranged in age from 6 weeks (after birth) to 70 years. Patient histories were reviewed to exclude known diseases of collagen. Guinea pig dermis was obtained from the pinna of $300-325 \mathrm{~g}$ male guinea pigs (5 animals). All dermal samples were stored for short periods $(0.5-1.5 \mathrm{hr})$ in cold, Hank's balanced salt solution, $\mathrm{pH} 7.3-7.4$. The loose subcutaneous connective tissue was removed from the sample prior to extraction. Tissues were cut into $4 \times 4 \times 1$ $\mathrm{mm}$ pieces prior to exposure to dissociative solvents.

Extraction procedures. Samples were extracted using two dissociative solvents; $8 \mathrm{M}$ urea in $0.3 \mathrm{M}$ Tris- $\mathrm{HCl}(\mathrm{pH} 8.6$ ) and $4 \mathrm{M}$ guanidine $\mathrm{HCl}$ in $0.05 \mathrm{M}$ Tris-HCl (pH 7.5). All extractions were carried out at $4^{\circ} \mathrm{C}$ and with continuous agitation. Tissue samples for ultrastructural observation were removed from the extraction solution at $30 \mathrm{~min}, 1,3$, and $5 \mathrm{hr}$.

Electron microscopy. The two principal fixatives used were $2.5 \%$ glutaraldehyde in $0.05 \mathrm{M}$ sodium phosphate buffer (pH 7.3) with $4 \%$ sucrose added and a mixture of $2.5 \%$ glutaraldehyde and $2 \%$ paraformaldehyde in $0.1 M$ sodium cacodylate ( $\mathrm{pH} 7.3$ ) containing $20 \mathrm{mM}$ calcium chloride. The same buffers were employed throughout the aldehyde and osmium tetroxide fixation sequences. Tissues were fixed for 2 to $3 \mathrm{hr}$ at room temperature, washed briefly in buffer and then exposed for $2 \mathrm{hr}$ to $1 \%$ $\mathrm{OsO}_{4}$ in the appropriate buffer. All tissue samples were stained en bloc with $2 \%$ uranyl acetate (14), the tissues immersed in $\mathrm{PO}_{4}$ buffer were extensively washed with cacodylate buffer (21) prior to exposure to uranyl acetate. All tissues were dehydrated through ascending percentages of ethyl alcohol beginning with a $50 \%$ concentration. Final dehydration was in propylene oxide prior to embedding in Epon-Araldite (17). Sections with silver-gray interference colors were cut with a diamond knife, collected on carbon reinforced, Formvar coated grids and stained with aqueous lead citrate and uranyl acetate in 50\% ethanol. A Philips EM 300 electron microscope was used to view the sections.

Determination of helical pitch. ${ }^{3}$ The planar or radial projection method of pitch calculation reported by Bouteille and Pease (6) was used. The fundamentals of this method are illustrated in Fig. 5a-e.

\section{RESULTS}

The effects of dissociative solvents on fibrillar structure. In untreated tissues,

${ }^{3}$ Pitch or gyre length as used in this paper is defined as the length, measured on the long axis of the cylinder, required for one full turn of the helix. longitudinally sectioned collagen fibrils appeared compact and demonstratd characteristic cross banding with a repetition occurring every $64 \mathrm{~nm}$. In cross section, collagen fibrils were compact, electron opaque structures; however, punctate sites of lead and uranium binding were usually observed.

Exposure of the dermis to both of the solvents studied resulted in the progressive expansion of the collagen fibrils. As this expansion and dissociation occurred, the helical substructure of the fibrils became apparent. At first not all fibrils were equally affected. After 30 min exposure to the solvents some longitudinally sectioned fibrils displayed acutely angled, spiral clefts (Fig. 1a). In cross section (Fig. 1b) these fibril clefts were observed to be surface manifestations of an internal, spirally oriented space.

Tissues sampled after $1 \mathrm{hr}$ showed further recruitment of fibrils into the expanded fibril population (Fig. 2a). Fibril expansion during these early periods varied not only between fibrils, but also within a single fibril. Greatly expanded regions (puffs) alternated with either only slightly "unwound" regions or essentially unaltered regions along the length of the fibril (Fig. 2b). By $3 \mathrm{hr}$ all fibers showed some degree of expansion (Fig. 3). After further dissociation $(5 \mathrm{hr})$, most fibrils appeared as indistinct units composed of a mass of microfibrillar aggregates (Fig. 4a), although, even at this time period some fibrils could be found which were only slightly expanded.

The effects of age on the stability of collagen fibrils in dissociative solvents. Collagen fibrils present in human dermal specimens from older individuals consistently resisted the effects of dissociative solvents better than those present in younger individuals. Comparisons between the extremes were most impressive. After $30 \mathrm{~min}$ in urea, collagen fibrils in a specimen taken from a 6 week old child were fully expanded, equal in degree to that observed 


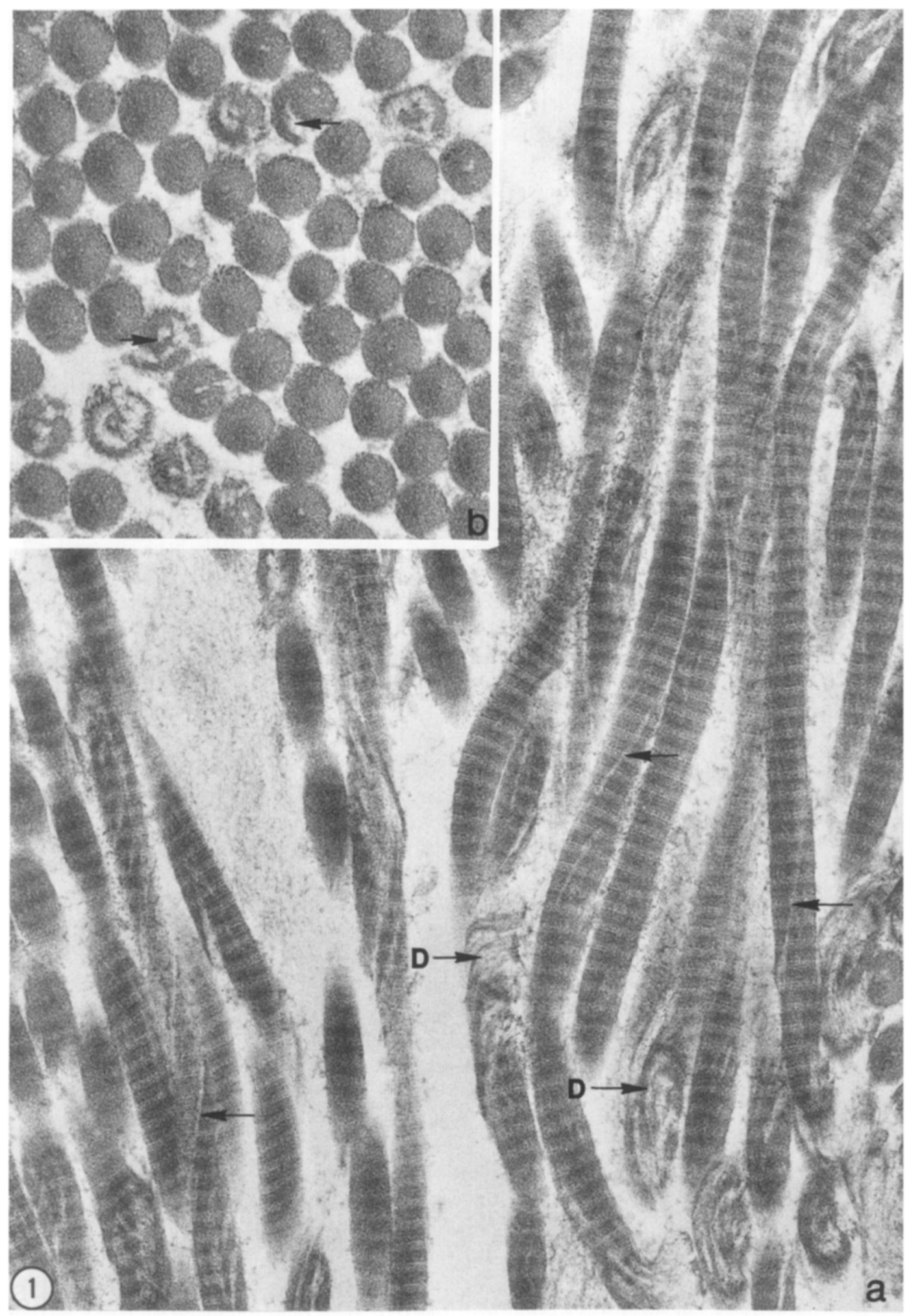

FIG. Ia. Diagonally oriented clefts (arrows) at the surface of dermal collagen fibrils are the first indication of fibril dissociation observed. Portions of a few fibrils (arrow "D") appear somewhat more dissociated. (b) The clefts in figure 1a are surface manifestations of an internal, spirally oriented space which is demonstrated (arrows) in these cross sectioned fibrils. A few fibrils demonstrate spaces, the openings of which are out of the plane of section. (a) $\times 49000$; (b) $\times 67000$. 

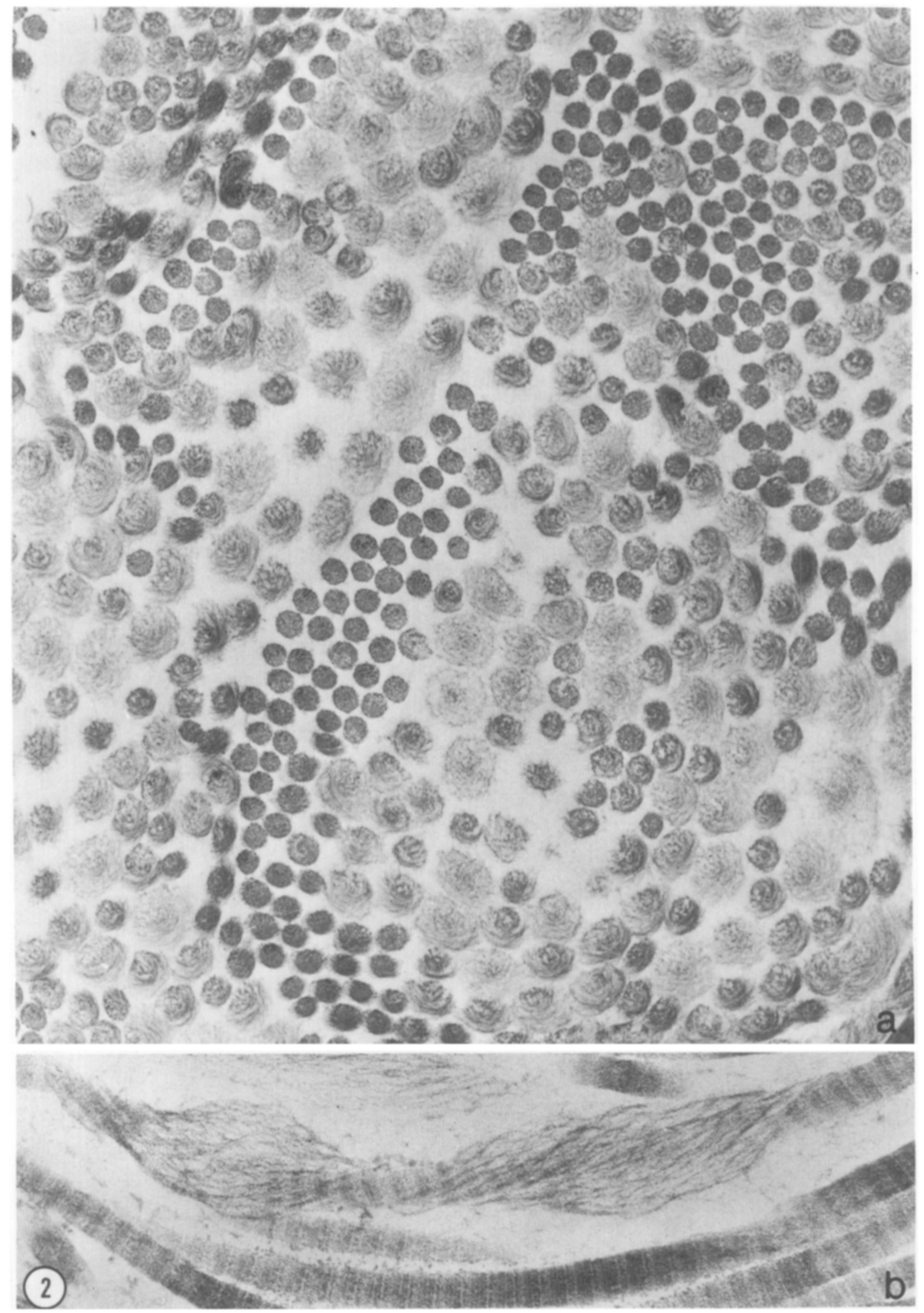

FIG. 2a. This micrograph illustrates the extent to which human adult, dermal collagen had expanded following $1 \mathrm{hr}$ in $8 \mathrm{M}$ urea. Various degrees of dissociation are depicted in this cross section of a collagen bundle. (b) As indicated here, regions of a single collagen fibril were often not equally expanded. Focal puffs, alternated with either intact or partly dissociated regions. (a) $\times 39000$; (b) $\times 67000$. 


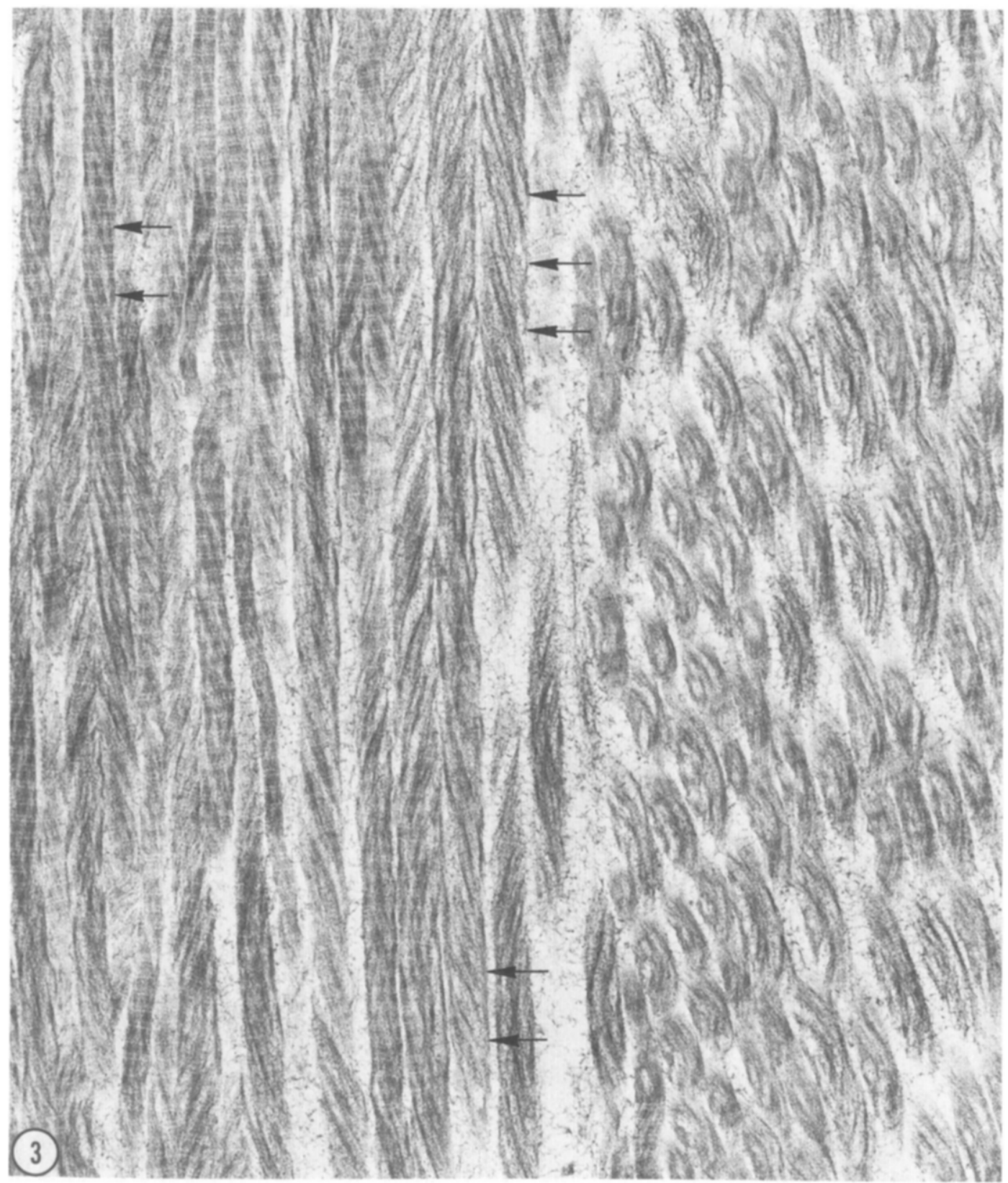

FIG. 3. This specimen of dermal collagen was exposed to urea for 3 hrs prior to fixation. Estimations of the pitch of the helices were made on collagen fibrils such as those illustrated in this micrograph. These fibrils demonstrate helical regions (arrows), cross banding and relatively distinct borders. The fibrils in the right portion of the micrograph were obliquely sectioned. Microfibrillar bundles in these obliquely sectioned regions appeared to be similarly oriented. $\times 36000$.

after $5 \mathrm{hr}$ (Fig. 4a). In contrast, samples taken from a 70 year old patient following $3 \mathrm{hr}$ in the same solvent showed only limited fibrillar expansion (Fig. 4b). This de- gree of variation was not observed in guinea pigs and probably relates to the fact that the age range of the animals examined was quite narrow. 

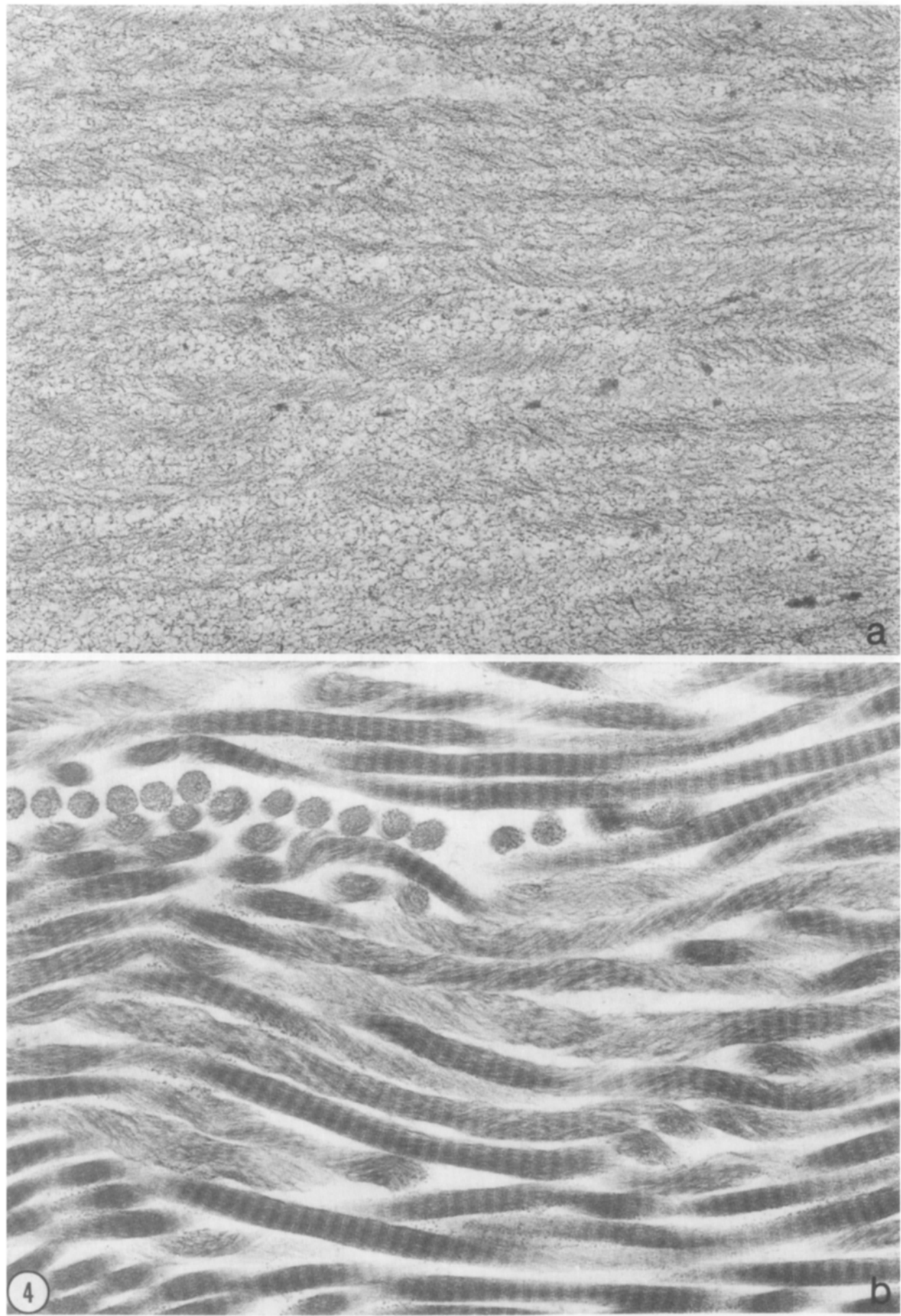

FIG. 4. These two micrographs depict the effect of age on fibril dissociation. (a) Following 30 min in urea the dermal collagen of a 6 week old child has uniformally dissociated into groups of microfibrils. (b) Following $3 \mathrm{hr}$ in urea the dermal collagen of a 70 year old man demonstrates limited fibril expansion. (a) $\times$ $36000 ;$ (b) $\times 36000$. 

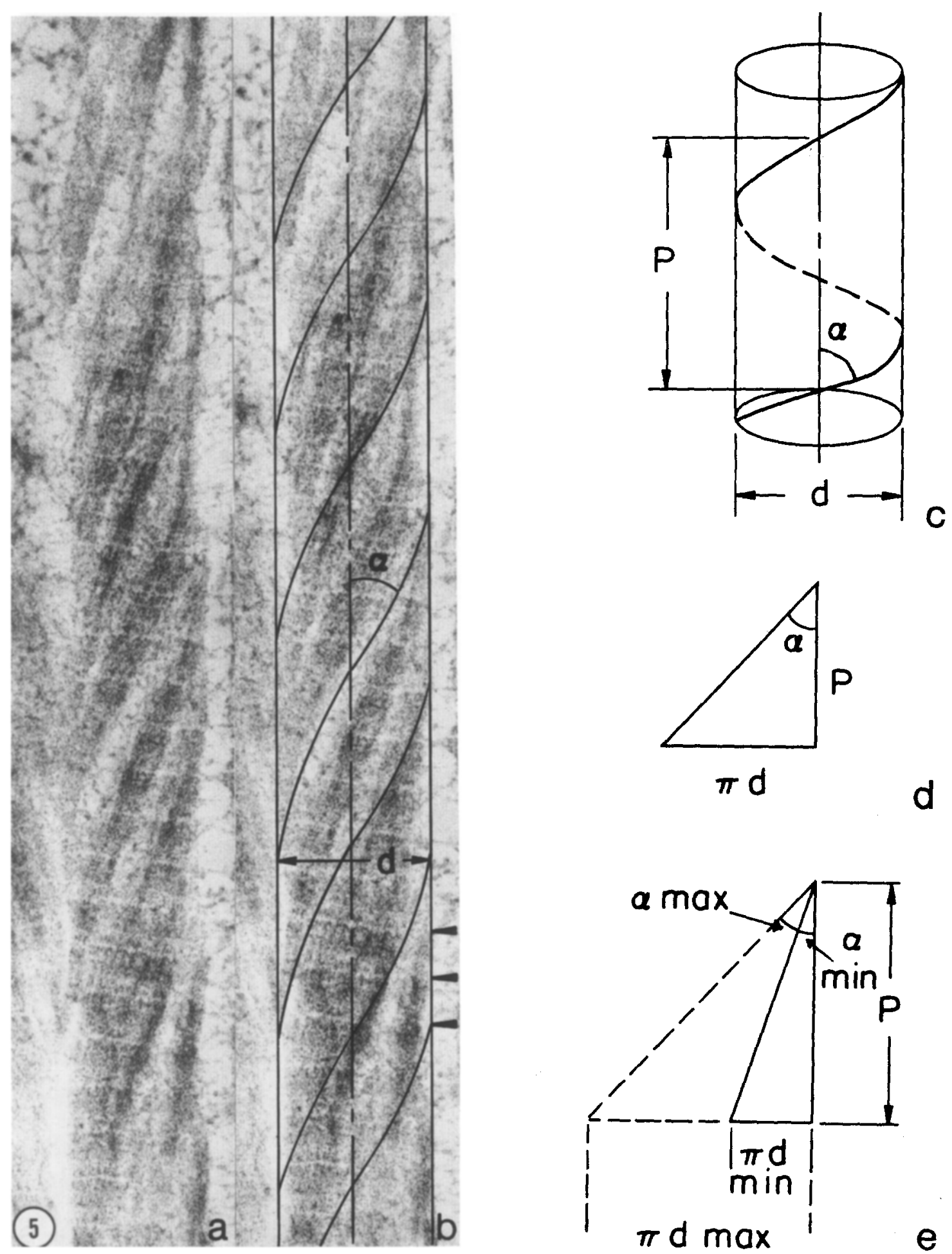

Fig. 5. This plate illustrates the method used for determining the pitch of the helix. (a) High power micrograph $(\times 120000)$ of a partially expanded fibril demonstrating helical substructure. (b) A micrograph of the same fibril with a superimposed drawing indicating how the diameter, $d$, and the angle of the spiral 
Characterization of the helix of the collagen fibril. Determination of the pitch or gyre length of the collagen fibril helix was made from measurements taken from areas in which the fibrils had similar diameters and exhibited similar degrees of expansion. These precautions were necessary because up to a 1.3 -fold variation in fibril diameter in "native" (nonexpanded) fibrils was observed in the tissues studied. The pitch both for populations of slightly expanded and fully expanded fibrils was $1.09 \pm 0.1 \mu \mathrm{m}$. This value is very similar to the pitch reported for rat aortic adventitia $(1.07 \mu \mathrm{m})(6)$. As expansion of the fibrils progressed, an increase in both fibril diameters and the values of $\alpha$ (Fig. $5 \mathrm{~b}$ and c) was measured. In Fig. 5e two planar projections based on average observed minimum and maximum values of fibril diameter and $\alpha$ are presented. Note that the measured values are so related that the pitch, $P$, remained the same for the two fibril populations. It appears that microfibrils slide axially on one another to adapt to the increased dimension of the expanded helix. Had such linear adjustments not have occurred, a common pitch value for expanded and nonexpanded fibrils would not have been achieved.

\section{DISCUSSION}

Initial attempts to interpret our findings were frustrated by what appeared to be the lack of any one common preparative step in those previous reports where a helical substructure in collagen fibrils had been observed. It finally became apparent to us that the collagen had always been exposed to various water-miscible liquids prior to being examined and that conventional "methylene bridge engendering fixatives" were not employed. Most frequently ethylene glycol or glycerol in 20-70\% (v/v) concentrations were used for extended periods of time (1 hr-several days) $(4,6,19)$. In Bouteille and Pease's study (6), the ethylene glycol treatment was followed by infiltration and embedment in hydroxypropyl methacrylate (HPM), which in monomeric form is quite hydrophilic. These authors noted that they saw the greatest degree of fibril expansion (and helicity) in glycol treated, HPM embedded tissue when compared with glycol treated and either Vestopal or Epon (less hydrophilic resins) embedded tissue. Less pronounced fibril expansion is apparently caused by the glycerol treatment routinely employed prior to freeze-fracturing.

It would appear that the mode of action shared by glycols and the urea-guanidinium class of denaturants is based on their ability to break hydrogen bonds. The solvent property of the polar, water-miscible glycols is based upon their ability to disrupt and form hydrogen bonds. The ureaguanidinium class of denaturants is believed to disrupt hydrogen and not covalent bonds (13). In light of the importance hydrogen bonding $(15,24)$, as well as ionic and hydrophobic interactions $(9,10)$, play in the various levels of lateral assembly of the collagen molecule-microfibril-fibril, it seemed that any agent capable of altering hydrogen bonds should have profound effects on the ultrastructural appearance of collagen. Further indication that hydrogen bonds are the site of action of dissociative solvents in collagen is provided by our observations made on mature and newly formed collagen. As collagen "matures" both inter- and intramolecular lysyl-derived, covalent, crosslinks develop which

substructure (direction of microfibrils) to the long axis of the fibril, $\alpha$, were determined. Arrows indicate the periodic cross banding which served as an inherent measure of magnification. (c) A diagram of a cylinder on the surface of which a helix has been drawn. The pitch of the helix, $P$, is indicated as well as $\alpha$ and $d$. (d) A planar projection of the surface of a cylinder indicating $\alpha$ and $P$. The base of the triangle, $\pi d$, is the circumference of the cylinder. The measurements thus related, when correlated with magnification, allow the calculation of the pitch of the helix. (e) Two planar projections based on measurements of $\alpha$ and $\pi d$ from maximally and minimally expanded fibrils superimposed upon one another demonstrate that a single, common pitch describes both helices. 
act to stabilize fibrillar structure previously maintained by hydrogen bonding ( 2 , 24). As reported in the Results section, collagen from adults resisted much more effectively the action of either guanidine$\mathrm{HCl}$ or urea than did the collagen present in the dermis of a recently born child.

The nature of fibril expansion observed following exposure of dermal collagen to the denaturants studied is unique and not like that which occurs during dissolution of dermal collagen by acetic acid (5). Nor does the expanded fibril resemble the loosely organized fibrils found in dermatosporaxis $(12,16)$. Furthermore, it appears that differences in structural organization may exist between collagen types; guanidinium chloride induced disaggregation of cartilage collagen does not reveal a helical substructure (1). The fibril expansion reported here suggests that as hydrogen bonding between microfibrils breaks down, the fibril follows a sequential pattern of dissociation. First large aggregates (bundles) of microfibrils are observed. These are subsequently subdivided into smaller and smaller aggregates as dissociation progresses. Though measurements of individual "microfibrils" were attempted, the size and variability encounted $(4.5-12.0 \mathrm{~nm}$ in diameter) suggested that complete dissociation of the fibril into a single class of microfibrils was not achieved.

In conclusion, we believe our findings strongly reaffirm those of previous investigations which reported that the collagen fibril was organized helically. This helical substructure can be demonstrated by using the urea-guanidine class of denaturants (prior to the formation of cross links by conventional aldehyde fixatives) which presumably disrupt hydrogen bonds. Further, it seems that intermolecular hydrogen bonding is of paramount importance in maintaining the structural integrity of the newly formed fibril. It now appears that the collagen fibril of the dermis, ad- ventitia, and tendon should be considered to be a helically organized structure in addition to the alpha chain, collagen molecule, and microfibril.

This study was supported by National Institutes of Health Grants DE 02731 and DE 03391. The authors are grateful to Dr. Harald Löe for his encouragement during the initial phases of this study and to Dr. Vincent C. Hascall for his continued interest in the work and critical review of this manuscript. They also wish to acknowledge the fine technical assistance of Mr. Phillip Singer.

\section{REFERENCES}

1. Anderson, H. C., and Sajdera, S. W., J. Cell Biol. 49, 650 (1971).

2. Bailey, A. J., Robins, S. P., ANd Balian, G., Nature (London) 251, 105 (1974).

3. Bairati, A., Petruccioli, M. G., and Torri Tarelli, L., in Fauard, P. (Ed.), 7th International Congress for Electronmicroscopy, Vol. 1, p. 635. Soc. Francaise de Microscopie Electronique, Paris, 1970.

4. Belton, J. C., Michaeli, D., and Fudenberg, H., Arthritis Rheum. 18, 443 (1975).

5. Borysko, E., in BREese, S. S., JR. (Ed.), 5th International Congress for Electronmicroscopy, Vol. 2, p. T-9. Academic Press, New York, 1962.

6. Bouteille, M., and Pease, D. C., J. Ultrastruct. Res. 35, 314 (1971).

7. Bruns, R. R., J. Cell Biol. 68, 521 (1976).

8. Bruns, R. R., Trelstad, R. L., and Gross, J., Science 181, 269 (1973).

9. Cunningham, L. W., Davies, H. A., and HamMONDS, R. G., JR., Biopolymers 15, 483 (1976).

10. Doyle, B. B., Hukins, D. W. L., Hulmes, D. J. S., Miller, A., and Woodhead-Galloway, J., J. Mol. Biol. 91, 79 (1975).

11. Doyle, B. B., Hulmes, D. J. S., Mrller, A., Parry, D. A. D., Piez, K. A., and WoodheadGalloway, J., Proc. Roy Soc. London B 186, 67 (1974).

12. FJølstad, M., and Helle, O., J. Pathol. 112, 183 (1974).

13. GoRDON, J. A., AND JENCKs, W. P., Biochemistry 2, 47 (1963).

14. Karnovsky, M. G., J. Cell Biol. 35, 213 (1967).

15. Miller, E. J., and Matukas, V. J., Fed. Proc. 33,1197 (1974).

16. O'Hara, P. J., Read, W. K., Romane, W. M., AND BRIDGES, C. H., Lab. Invest. 23, 307 (1970).

17. Palevitz, B. A., ANd Newcomb, E. H., J. Cell 
Biol. 45, 383 (1970).

18. Piez, K. A., ANd Miller, A., J. Supramol. Struct. 2, 121 (1974).

19. RAyns, D. G., J. Ultrastruct. Res, 48, 59 (1974).

20. REED, R., WOOD, M. J., AND KEECH, M. K., Nature (London) 177, 697 (1956).

21. Simionescu, N., Simionescu, M., And Palade,
G. E., J. Cell Biol. 53, 365 (1972).

22. SmrTh, J. W., Nature (London) 219, 157 (1968).

23. Szirmori, J. A., Van RaAmsdonk, W., GalavaJi, G., J. Cell Biol. 47, 209a (1970).

24. Tanzer, M. L., Science 180, 561 (1973).

25. WaIsman, J., AND Smith, D. W., Cancer 22, 671 (1968). 